\title{
Integration of a Real-Time Node for Magnetic Perturbations Signal Analysis in the Distributed Digital Control System of the TCV Tokamak.
}

\author{
C. Galperti, S. Coda, B. P. Duval, X. Llobet, P. Milne, O. Sauter, J. M. Moret, D. Testa and the TCV team
}

\begin{abstract}
This paper presents the integration of an acquisition and computing unit capable of acquiring and processing fast magnetic signals in real-time in the control system of the TCV tokamak. All aspects of system integration and testing are reported, leading to testing of the system on plasma discharges. An example of a real-time analysis algorithm designed for detecting and classifying NTM plasma instabilities is also described.
\end{abstract}

Index Terms-Automatic detection of tearing modes in fusion plasmas, real-time Tokamak control systems, real-time signal analysis systems.

\section{INTRODUCTION}

$\mathbf{T}$ $\mathrm{HE}$ analysis of fast magnetic signals measured by magnetic probes installed on a tokamak vessel has been widely employed to detect and analyze a number of plasma instabilities, notably rotating tearing modes. The high frequency components of the measured signals contain information directly related to rapid changes within the plasma, for instance those generated by tearing mode magnetic islands rotating in the torus. Off-line analysis of these signals employs powerful mathematical tools such as spectrograms, principal component analysis and amplitude-phase fitting algorithms [1] [2] [3] [4]. These provide copious information on magnetic islands topology, size and evolution.

In this paper we describe the integration of these techniques into the TCV digital real time control system to execute advanced magnetic analysis codes during the tokamak discharge. The final goal is to provide plasma health status information to decision making algorithms that can initiate actuator reactions. Severe design constraints, posed by TCV's real-time environment, had to be addressed. TCV presently employs a distributed real time control system capable of controlling the entire plant during a discharge, consisting of commercial PCs interconnected with a self controlled high speed fiber optic digital link [5] [6] [7]. Each node of the system can house ADC and DAC boards that interact with the plant.

This paper describes the integration of a supplementary realtime magnetic coil analysis node into this system, taking into

Manuscript received May 30, 2016; revised January 26, 2017.

C. Galperti, S. Coda, B. P. Duval, X. Llobet, O. Sauter, J. M. Moret and D. Testa are with Swiss Plasma Center (SPC) of the Ecole Polytechnique Fédérale de Lausanne (FPFL). CH-1015 Lausanne, Switzerland. Contact email: cristian.galperti@epfl.ch

P. Milne is with D-TACQ Solutions Ltd. James Watt Building, Scottish Enterprise Technology Park, East Kilbride, G75 0QD, Scotland UK. consideration the restrictions posed by the demanding realtime environment whilst respecting all the pre-existent features of the control system. It is organized as follows: section II introduces the TCV distributed control system, section III describes the integration of the new node and finally section IV presents system tests during TCV operation.

\section{The TCV Distributed Digital Control System}

The TCV tokamak has a fully functional digital real time control system capable of controlling almost all aspects of a plasma discharge. The system is based on a real-time sharing data network (reflective memory) of modular computer nodes, each an embedded or desktop PC which may include local ADC and/or DAC cards. Owing to the restricted resources, particularly in terms of manpower, available to the TCV team, design choices were made favoring simplicity, flexibility and maintainability. The main requirements and resulting design choices are listed in Table I. From the top level, the system is a distributed acquisition and processing real-time system build on top of standard COTS PCs augmented with ADC and DAC boards and high speed dedicated communication links. Hence its French name: Système de Contrôle Distribué (or SCD), which translates into Distributed Control System.

\section{A. Real-time computer nodes}

Figure 1 shows the SCD control system layout with the connectivity to the diagnostics and actuators. Some nodes are connected to a compact-PCI (cPCI) crate hosting one or more D-tacq ACQ-196 acquisition cards with 96 ADCs, and output cards housing 16 or 32 DACs. Some nodes are only connected via the reflective memory network and act as computational nodes. At present there are seven nodes, the seventh is the one described in this work.

All the nodes exchange data with all the others exploiting the reflective memory link, (RFM in brief) an industry standard high-speed digital communication system that transparently synchronizes a shared memory to all nodes [8]. The synchronization is performed without user and/or kernel intervention but it has the drawback that no data handshaking is performed so the user is responsible of preventing read-write race conditions. In SCD this is accomplished by using a node as a RFM master which synchronizes all operations on the RFM of all others nodes w.r.t. a common timebase. On each clock of the RFM master node (this is assigned by the SCD operator), to prevent read/write collisions, we alternate a read or write 
TABLE I

REQUIREMENTS AND RESULTING DESIGN CHOICES FOR THE TCV DISTRIBUTED DIGITAL REAL TIME CONTROL SYSTEM

\begin{tabular}{|c|c|c|}
\hline Aspect & Requirement & Design choice \\
\hline Hardware & $\begin{array}{l}\text { Minimise learning curve for new hardware, minimise } \\
\text { spares, cost }\end{array}$ & $\begin{array}{l}\text { Base system on same ADC modules (D-tacq ACQ-196, } \\
\text { cPCI form factor) already used for many TCV } \\
\text { diagnostics. Use COTS Linux PCs. }\end{array}$ \\
\hline $\begin{array}{l}\text { Sampling } \\
\text { rate }\end{array}$ & $\begin{array}{l}1 \mathrm{kHz} \text { (shape control) to } 250 \mathrm{kHz} \text { (MHD mode } \\
\text { detection), } 100 \mathrm{kHz} \text { (vertical stabilisation) }\end{array}$ & $\begin{array}{l}\text { Use different nodes that can run at different sampling } \\
\text { rates, vertical stabilisation ( } 1 \text { channel) still handled by } \\
\text { the analog system. }\end{array}$ \\
\hline $\begin{array}{l}\text { Real-time } \\
\text { performance }\end{array}$ & Hard real-time performance on COTS Linux PC & $\begin{array}{l}\text { Standard Linux OS with interrupt masking during } \\
\text { real-time code execution, progressive migration to } \\
\text { multi-core processors and SMP kernels capable of } \\
\text { reserving cores for real-time processes. }\end{array}$ \\
\hline Diagnostics & $\begin{array}{l}>200 \text { diagnostic channels (100+ magnetics, } 64 \text { soft } \\
\text { X-ray, } 14 \text { interferometer...) plus flexibility to add more } \\
\text { in the future. }\end{array}$ & $\begin{array}{l}\text { ACQ-196 ADC front-ends with } 96 \text { channels each, } \\
\text { maximum of } 3 \text { cards per node used so far. }\end{array}$ \\
\hline Actuators & $\begin{array}{l}\text { Control all TCV actuators including } 16 \text { PF coils, } 7 \text { EC } \\
\text { launchers, } 6 \text { EC power supplies, } 4 \text { gas valves, with } \\
\text { possibility for expansion. }\end{array}$ & $\begin{array}{l}\text { DAC modules with up to } 32 \text { channels each, possibility } \\
\text { to connect different nodes to different actuators. }\end{array}$ \\
\hline $\begin{array}{l}\text { Inter-node } \\
\text { communica- } \\
\text { tion }\end{array}$ & $\begin{array}{l}\text { Transparent, low overhead and kernel-independent } \\
\text { communication between different nodes of the control } \\
\text { system. }\end{array}$ & $\begin{array}{l}\text { Reflective memory system (from General Electric) with } \\
\text { reserved memory space for each node. }\end{array}$ \\
\hline Algorithms & $\begin{array}{l}\text { Rapid algorithm development, debugging, simulation, } \\
\text { sharing, archiving and tuning during operations }\end{array}$ & $\begin{array}{l}\text { Real-time programming entirely with Simulink } \\
\text { block-programming language, automatic conversion to } \\
\text { C. }\end{array}$ \\
\hline Data storage & $\begin{array}{l}\text { Algorithms and data must be stored for later analysis, } \\
\text { troubleshooting }\end{array}$ & $\begin{array}{l}\text { Real-time input-output data stored to MDSplus } \\
\text { database, operative code folder under SVN version } \\
\text { control and committed at every shot. }\end{array}$ \\
\hline
\end{tabular}

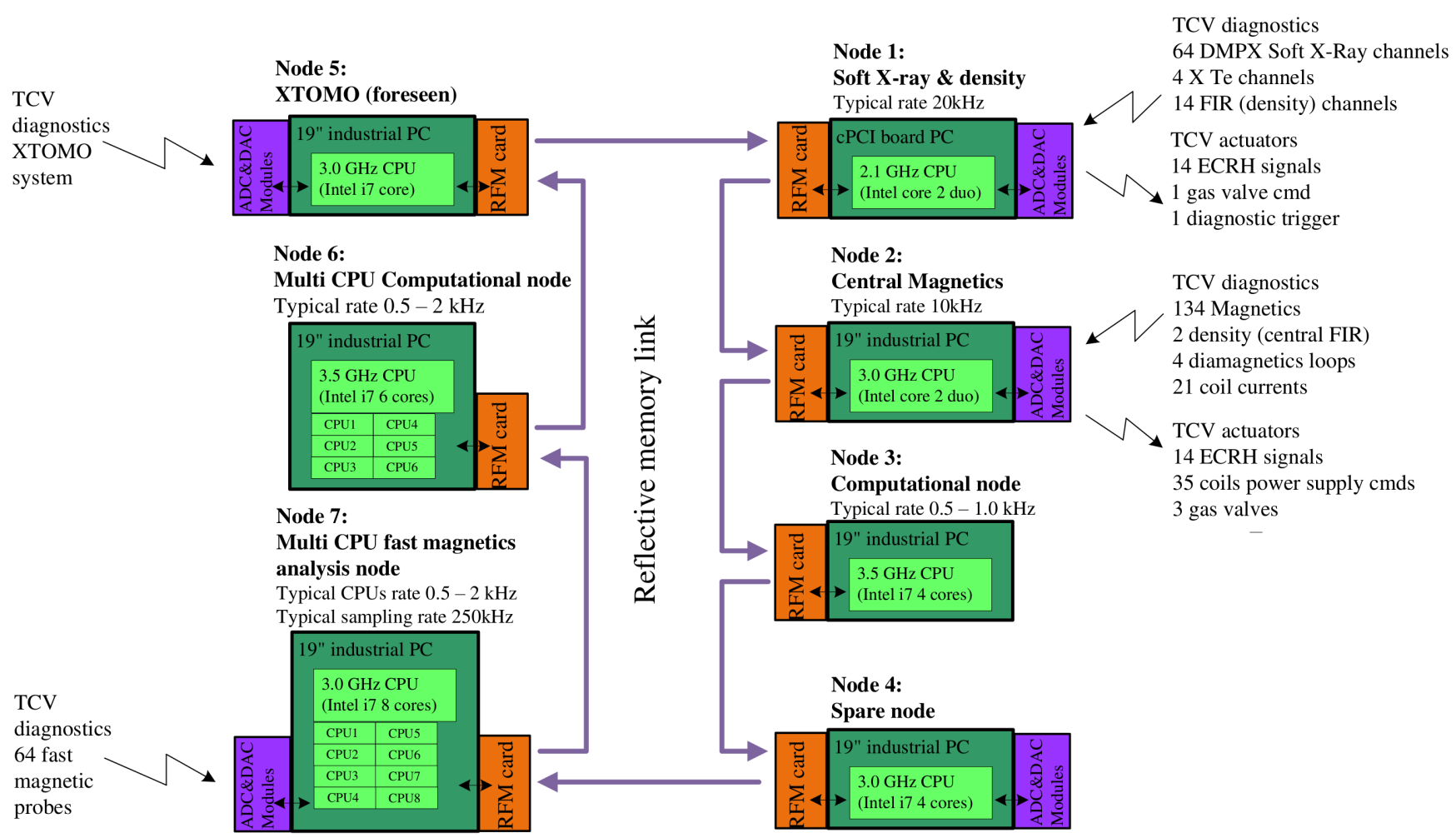

Fig. 1. Top view of the TCV Système de Contrôle Distribué.

from/to the RFM using DMA (i.e. write on odd cycle and then read on even cycle). All nodes must be synchronized with the write and read cycle, and all the nodes write (or read) at the same time, although nodes may skip cycles depending on their control rates [7].

The nodes with ADCs and DACs are interfaced to machine's 
diagnostics systems and to machine's actuators. Node 1 is interfaced to 2 soft-X diagnostics (DMPX, or Duplex Multiwire Proportional soft X-ray counter, a pinhole type soft$\mathrm{X}$ camera and $\mathrm{X}-\mathrm{Te}$, a four filter soft-X spectrometer that provides central electron temperature using the differential filter method). It is also interfaced to the 14 vertical chords of the Far InfraRed (or FIR) interferometer providing the electron density profile information. Node 2 acquires all magnetics measurements from the tokamak and is thus responsible for plasma shape and position control; it also acquires the central FIR channel for real-time control of the density. This node is used routinely as the main plasma position and density controller and it is almost always the RFM master node. Node 3 is a computational node that computes plasma magnetic equilibrium in real-time. Node 4 is a replacement node for node 2, while node 5 is an acquisition and processing node connected to the 200 channel soft x-ray tomographic system. Node 6 is a very recently installed multicore computational node that has been used to run multicore complex control codes (a faster real time equilibrium reconstruction replica and RAPTOR based advanced plasma performances controllers). Finally, node 7 is devoted to real-time analysis of fast magnetic perturbations in the plasma.

\section{B. Software organisation}

Fig. 2 presents the modular, portable software organization of the SCD control system. The SCD code is divided into two main sections:

1) Hardware interface code written in $\mathrm{C} / \mathrm{C}++$ language by the system developers, it provides the input/output interface to the control algorithm code. Once compiled, the executable is uploaded to real-time computer nodes, where it is considered fixed and unchanging between plasma shots. However, the hardware code can change its functional behaviour depending on external configuration parameters, such as a varying operational mode with or without suspending interrupts, the number of ADC/DAC cards, number of cores to be used (for multicores nodes), the data shared via RFM memory, etc.

2) Control algorithm code realised in MathWorksSimulink [9] block programming language by the control algorithm author(s), using Simulink templates given by the system developers. It performs signal processing and computational actions to provide output signals consisting of new values for the actuators, the reflective memory and other signals (probe signals) used for post-shot analyses. This algorithm is in user-friendly Simulink block format and is automatically converted into target code that is a dynamically linked shared object library by MathWorks Simulink Embedded Coder (SEC) [10].

This modular architecture offers great advantages. The abstraction from the hardware specific code makes the control algorithm code portable, i.e. control algorithms tested on TCV could be readily reused in other fusion facilities with minor adaptations. Another salient advantage of this modularity is its flexibility, i.e. the control algorithm can be developed on any computer equipped with Matlab-Simulink without requiring the Simulink Embedded Coder (SEC) package installed, and then simulated for debugging using the real diagnostic signals of past shots. This feature has already been exploited by external collaborators from several institutions across Europe and beyond to test their control algorithms on TCV. Only once the correctness and the robustness of the control algorithm are verified in simulation using experimental data of the previous shots, is it commissioned into RT system. Another essential advantage is that algorithm authors can use the extensive Simulink block library for standard components such as filters, integrators, matrix multiplications and other advanced signal processing tools. For TCV, standard custom-built blocks are provided for interfacing algorithms with TCV diagnostics and actuators, implementing calibration factors, signal selection, actuator constraints and saturation, etc.

\section{INTEGRATION OF NODE 7}

Having introduced the general architecture of TCV SCD control system, now we explain the integration of the new node 7 .

\section{A. Hardware and the hardware interface code}

From the point of view of the hardware architecture, node 7 is similar to all others SCD nodes, being constituted of a cPCI acquisition crate fitted with a DTACQ ACQ196 96 channels synchronous digitizer card, a rear transition module (DTACQ RTM type T) and an industrial PC, acting as the host processing unit. The industrial $\mathrm{PC}$ is fitted with a Reflective Memory Card of the same kind as all the other nodes of the system to communicate with real time network.

As mentioned, node 7 is devoted to fast magnetic signal acquisition and processing in real time. The main technical requirements to be fulfilled are as follows:

1) Sampling frequency. The frequency of the usually observed plasma instabilities of the TCV tokamak lie in the range from some hundreds of $\mathrm{Hz}$ to $100 \mathrm{kHz}$. Furthermore, phase coherence based algorithms are often used to infer coherent plasma structures and so simultaneous sampling of signals is mandatory. Simultaneous sampling of at least 32 magnetic signals at frequencies above $200 \mathrm{ksps}$ is required.

2) CPU process time. The previous requirement limits the CPU processing time since higher sampling rates lower the available processing time on the CPU (being equal to the sampling period). The aim of this node is to execute complex analysis algorithms on high speed multichannel data streams within a reasonable processing time.

3) Multi-processing capability. Processing algorithms may be spread over multiple cores to increase the computational power.

4) Legacy RFM interface. Data sharing with other nodes of the control system have to comply with the existing data distribution scheme described in II-A.

5) Processing algorithms written in Mathworks/Simulink. The analysis algorithms should 


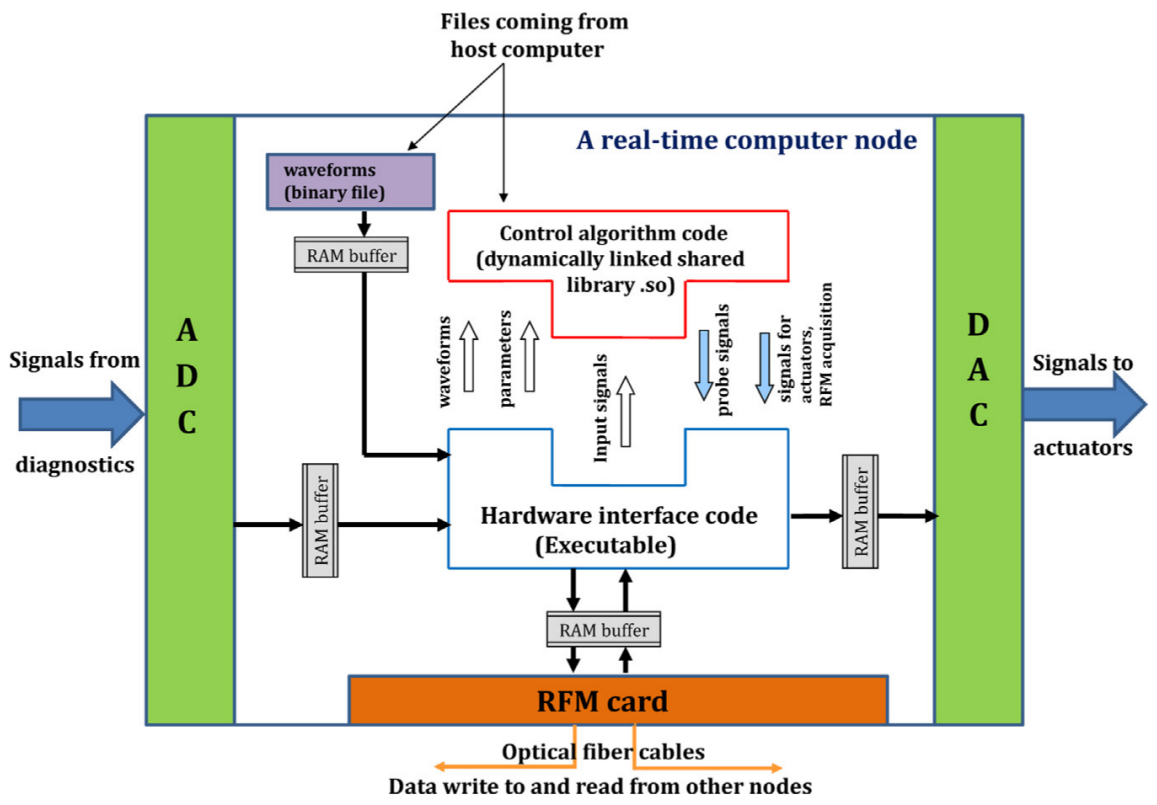

Fig. 2. Software organization in each node.

be developed in Matlab/Simulink and automatically uploaded to the analysis node. Therefore, node 7 must be fully compatible with the software architecture described in figure 2 .

To comply with the above requirements, we adopted a packet acquisition-processing scheme where the ADCs have a double buffered data path allowing the CPUs to operate on each data packet as described in [11]. The acquisition hardware employs an ADC subsystem with a D-TACQ ADC196 board augmented with a RTM-T rear transition module [12]. This module provides a high speed PCI-Express 1x link to the host $\mathrm{PC}$, which is equipped with a recent, high-end, motherboard hosting an Intel i7-5960X 8-core 3GHz processor. The adopted kernel is Scientific Linux 6.7 especially configured for usermode RT capabilities with a dedicated hardware interface code fully compliant with fig. 2 . The ad-hoc configured kernel together with hardware interface code exploit the multicore CPU by distributing tasks on the cores as summarized in table II. The kernel is restricted to the first core by Linux init command options flexibility with all main kernel threads and user processes executed on this core leaving the others free for user real-time processes and/or threads; the only kernel threads remaining on these cores are the basic Linux kernel threads required for handling the cores (such as the interrupt handling kthread, the migration kthread and the watchdog kthread).

Once the system enters the real-time state before the plasma discharge, the hardware interface code is launched as an user process on core 2. Node 7's hardware interface code is a multi-thread process that spawns a thread per used core to distribute the computational tasks. Besides hosting the hardware interface code, core 2 receives data-ready interrupts from the acquisition subsystem (which thus also synchronizes the node), distributes fresh ADC data to the processing threads and prepares RFM read/write buffer (without actually moving them
TABLE II

TASK ASSIGNMENT TO CPU CORES OF SCD NODE 07

\begin{tabular}{|l|l|}
\hline Core no. & Task \\
\hline 1 & $\begin{array}{l}\text { Linux kernel (forced here in the boot command). } \\
\text { Data communications with DTACQ196/RTM-T } \\
\text { acquisition subsystem, data dispatch to processing cores. } \\
\text { Processing core } 1 .\end{array}$ \\
4 & $\begin{array}{l}\text { Processing core } 2 . \\
\text { Processing core } 3 .\end{array}$ \\
6 & $\begin{array}{l}\text { Processing core } 4 . \\
\text { Synchronization handshaking and data exchange on the } \\
8\end{array}$ \\
\hline
\end{tabular}

on the RFM board). Cores 3 to 6 each can host a processing Simulink algorithm, making this node fully multiprocessing. Finally core 7 is used to synchronize data transmission and reception on the RFM card; we employ a dedicated thread on a dedicated core to run this task since the node must resynchronize itself to the master RFM node's read and write clocks and the fastest way to achieve this is by polling the RFM card. Since a polling thread usually consumes all the core activity rate and can only be preempted by an higher priority thread (on a RT kernel), we chose to employ a separated core and thread to do this, leaving core 2 fully committed to real time ADC data handling.

Figure 3 presents the typical working time chart of node 7. The ADCs on the ACQ196 board are clocked at $256 \mathrm{kHz}$ synchronous with TCV main clock at $1 \mathrm{MHz}$; the synchronous sampling clock is generated by a high accuracy DDS (Direct Digital Synthesizer) chip on a companion board in the acquisition cPCI crate. ADCs' data stream is directed to a double 
buffer on the acquisition board, when one buffer is full, a data ready interrupt is sent to the host PC and the buffer is switched, releasing the just filled one to the host CPU. The arrival rate of this interrupt is evaluated as follows:

$$
f_{\text {int }}=\frac{f_{s}}{N_{s c h}}
$$

where $f_{\text {int }}$ is the interrupt frequency, $f_{s}$ the sampling frequency and $N_{s c h}$ the number of samples per channel in the buffer. With typical working parameters: $f_{s}=256000$ and $N_{s c h}=256$ the interrupt frequency is $f_{\text {int }}=1000$, i.e. we have a $1 \mathrm{~ms}$ cycle time. With this hardware, $N_{s c h}$ must be a power of two, hence the choice of $256 \mathrm{kHz}$ for the sampling frequency to obtain a $1 \mathrm{~ms}$ cycle time.

The hardware interface code exploits Intel TSC (Time Stamp Counter [13]) technology to precisely time stamp its activities. Every core has a 64 bit wide counter clocked at the CPU nominal frequency that can be read very quickly using dedicated assembler code. These counters can be exploited to time stamp key passages of the hardware interface code, providing the timing information for system monitoring and algorithm benchmarking. The initial timing information is the measured system cycle time, i.e. time interval $\mathrm{t} 1$ in figure 3 . The time trace of this value is the first indicator of good or improper RT behavior of the system; with the working parameters introduced in the previous paragraph, this trace should be a constant value of $1 \mathrm{~ms}$. Once the data ready interrupt has been issued, the CPU must copy fresh ADC data from the acquisition board to main memory, distribute it to the processing cores and update the RFM buffers. This requires time that is stored in time stamp $t 2$ and is depicted as the purple phase in figure 3. After this phase the processing cores have new data to process and thus processing algorithms can be triggered; several processing cores act on ADC data in parallel as depicted by the "CPU 1 processing" and "CPU 2 proc" phases in figure 3 , their processing time are stored in markers $t 5$ and t6. Up to four processing cores can be used: the algorithm presented in this paper uses two. The available computational time to all processing cores in every cycle is equal to $\mathrm{t} 1$ - $\mathrm{t} 2$. The hardware interface code can allow some processing cores to work at a lower speed w.r.t. the main ADC data ready by triggering at a fraction $1 / \mathrm{N}$ of the rate of data ready interrupt arrival; this allows these cores to operate at lower speed w.r.t. data packet rate, granting more computational time at the expense of analyzing only a subset of the signals windows, i.e. one every N. In this case data exchange with other cores happens every $\mathrm{N}$ computing cycle as well. Following the $\mathrm{t} 2$ phase, the hardware interface code exchanges data with the reflective memory board. It must first synchronize itself with the read and write phases dictated by the RFM master node. Time elapsed from the data ready interrupt and the read and write synch time are recorded in time stamps $\mathrm{t} 3$ and $\mathrm{t} 4$ of figure 3 .

All timing information are stored to the SCD MDSplus tree and are used to check the node functionality.

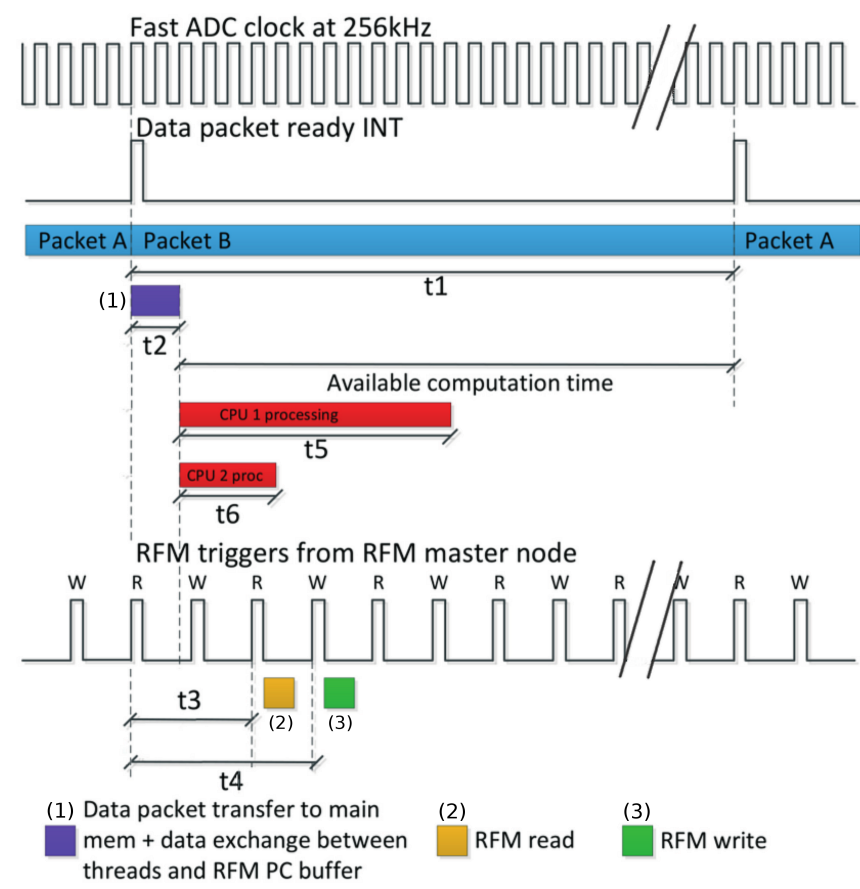

Fig. 3. Time chart of node 7 cycle (relative time scale between clocks is only reported qualitatively).

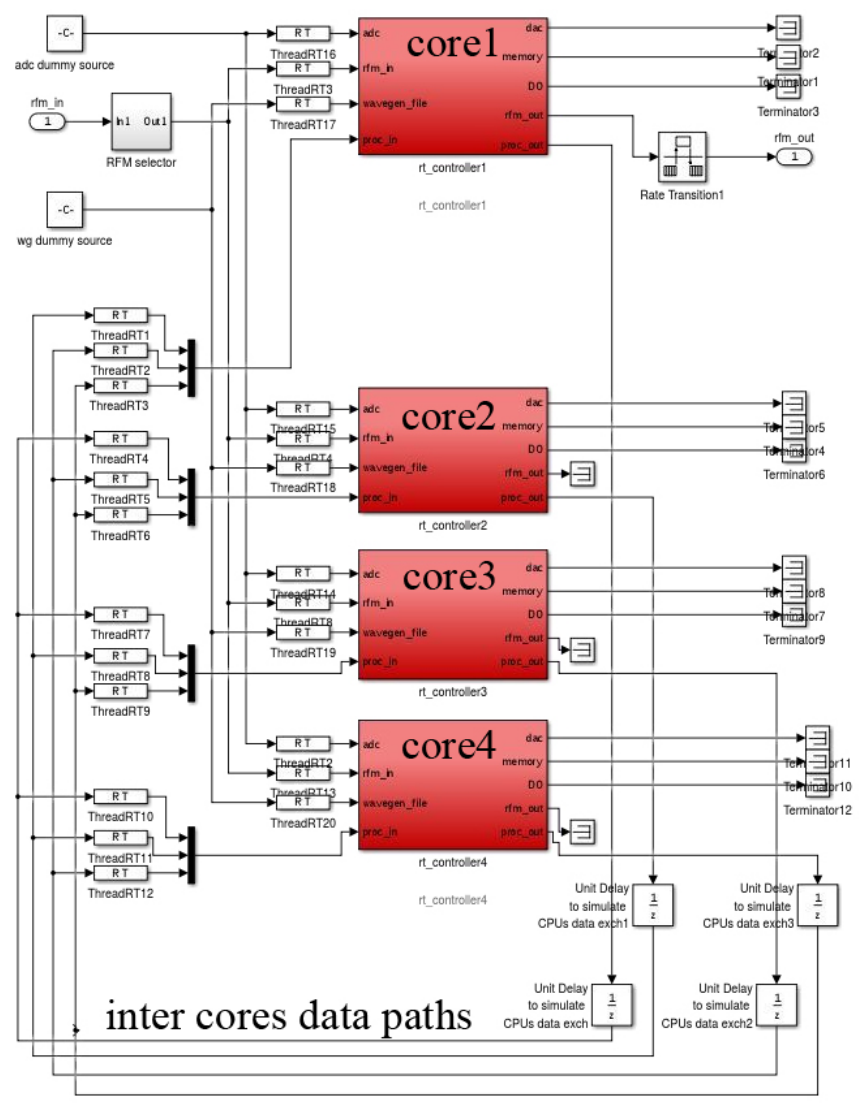

Fig. 4. Top Simulink diagram of node 7.

\section{B. Software, the Simulink model and integration of the MHD analysis code}

As all the other nodes of the control system, the control code (or diagnostic processing code, in this case) is written 


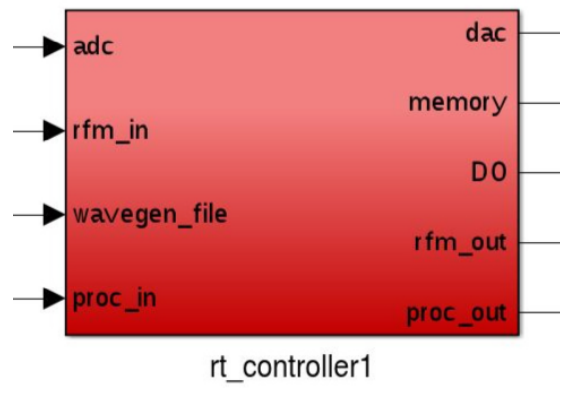

Fig. 5. Simulink diagram of one of node 7 processing cores.

as a Simulink block diagram. This enormously facilitates algorithms' management during both experimental activity and later data analysis. Since node 7 is a multicore processing machine, a dedicated Simulink block model was developed. This model is shown in figure 4. Every processing core is modeled as Simulink block with standardized input and output ports; since node 7 has 4 parallel processing cores, four of these blocks are inserted. Each block represents an independent processing thread that can process input data in parallel w.r.t. the others.

Once one TCV pulse is initiated, the active blocks' content is opened by the SCD build process, a Linux shared object library is generated for each and these libraries are distributed to node 7. Upon entering the real-time phase of the discharge, the hardware interface code of node 7 is launched and loads these shared objects assigning their processing code to one thread per core. During the discharge, these cores process data from the fast magnetic signals in parallel and return the results via the RFM real time network, as described in III-A.

Figure 5 shows a Simulink block representing a single core. This is the Simulink object that is then translated into $\mathrm{C}$ code and then compiled into the Linux shared library upon shot preparation. It has four input and five output ports whose meaning and sizes are summarized in table III. This block acts as a wrapper for every processing algorithm, a Simulink model inserted and it also provides a clear and straightforward mean of standardizing the input and output interfaces.

Figure 4 shows the actual interconnections of the core models on the node CPU. Basically every core's proc_out port is looped back to the proc_in port of the other cores with one cycle delay. This provides a simple and general modeling of inter-thread data communication. The hardware interface code takes care of these data movements once the algorithms models are uploaded to node 7. Obviously, this is not the most efficient way to handle inter core data communications since a fixed amount of data (figures are in table III) is always exchanged irrespective of what is really present on the proc_out ports. Nevertheless, we chose a general, fixed interface, approach at the expense of communication overhead. This is also true for all the input and output ports.

Input and output ports to and from the CPU are arranged with the twofold aim of mimicking the real system (particularly the hardware interface code) allowing SCD re-simulation on a past data in Simulink. As an example, the ADC input port is connected to a InputFromWorkspace block that is initialized
TABLE III

INPUT AND OUTPUT PORT MEANING OF THE EMPLOYED SIMULINK MODEL OF 1 PROCESSING CORE

\begin{tabular}{|c|c|c|}
\hline $\begin{array}{l}\text { Port } \\
\text { name }\end{array}$ & Function & Size \\
\hline adc & $\begin{array}{l}\text { data buffer from the ADCs, it contains } \\
\text { the last acquired data packet. }\end{array}$ & $\begin{array}{l}16384 \text { words } \\
\text { (16 bits) }\end{array}$ \\
\hline rfm_in & $\begin{array}{l}\text { RFM buffer from other nodes, it contains } \\
\text { the last data sent of the RFM network by } \\
\text { other nodes. }\end{array}$ & $\begin{array}{l}1024 \text { singles } \\
\text { (32 bits) }\end{array}$ \\
\hline $\begin{array}{l}\text { wave- } \\
\text { gen_file }\end{array}$ & $\begin{array}{l}\text { It can be used to provide pre computed } \\
\text { signals in alternative of putting them into } \\
\text { the Simulink model. }\end{array}$ & $\begin{array}{l}1024 \text { singles } \\
\text { (32 bits) }\end{array}$ \\
\hline proc_in & $\begin{array}{l}\text { Inter-cores data communication input, it } \\
\text { provides the mean of receiving data from } \\
\text { other cores of the CPU. }\end{array}$ & $\begin{array}{l}1536 \text { singles } \\
\text { (32 bits) }\end{array}$ \\
\hline dac & $\begin{array}{l}\text { DAC output port, it contains data to be } \\
\text { sent to node's DACs, if present. }\end{array}$ & $\begin{array}{l}96 \text { words } \\
\text { (16 bits) }\end{array}$ \\
\hline memory & $\begin{array}{l}\text { Real-time storage memories. Data put } \\
\text { into this port is stored into dedicated } \\
\text { channels during the discharge and stored } \\
\text { to MDSplus database, i.e. it is the main } \\
\text { way to check and debug the real-time } \\
\text { code. }\end{array}$ & $\begin{array}{l}256 \text { doubles } \\
\text { ( } 64 \text { bits) }\end{array}$ \\
\hline DO & $\begin{array}{l}\text { Digital output port, it contains data to be } \\
\text { sent to node's digital outputs, if present. }\end{array}$ & $\begin{array}{l}8 \text { int }(32 \\
\text { bits) }\end{array}$ \\
\hline rfm_out & $\begin{array}{l}\text { RFM write buffer, this is the port used to } \\
\text { transmit data on the RFM network; data } \\
\text { put here by the algorithms are broadcast } \\
\text { on the RFM to other nodes. }\end{array}$ & $\begin{array}{l}196 \text { singles } \\
\text { (32 bits) }\end{array}$ \\
\hline proc_out & $\begin{array}{l}\text { Inter-cores data communication output, it } \\
\text { provides the mean of transmitting data to } \\
\text { other cores of the CPU. }\end{array}$ & $\begin{array}{l}512 \text { singles } \\
(32 \text { bits })\end{array}$ \\
\hline
\end{tabular}

with previous shot ADC data, taken from the SCD MDSplus database if the model is used for re-simulation. It is connected to the real ADCs by the hardware interface code when the core model is uploaded to node 7 . The same happens for the other ports, allowing an efficient and easily manageable re-simulation of past shots whilst providing a very flexible processing algorithm development environment.

Node 7 was programmed and tested with the SVD rt MHD analysis code described in [14]. In essence this code employs a Singular Value Decomposition of a matrix whose columns are a bandpass filtered copy of the fast magnetic signals followed by a post-processing phase that compares the experimental principal axes (i.e. the columns of one of the three matrices computed by the SV decomposition) with those computed on a numerically generated set of signals from a theoretical model of rotating modes. This algorithm was ported to the Simulink representation introduced here. It exploits the multiprocessing capability of node 7 operating on to 2 cores: the first computes the SVD decomposition of live signals from the ADCs and the second computes the SVD decomposition of the theoretical model. Not only does this reduce the computational time but the theoretical model can be made dependable on other live plasma signals. In the presented implementation it depends upon the plasma magnetic axis in the machine. Conventional oddN and evenN MHD markers have also been included in the analysis code.

Figure 6 reports the algorithm that runs on core 1. The 


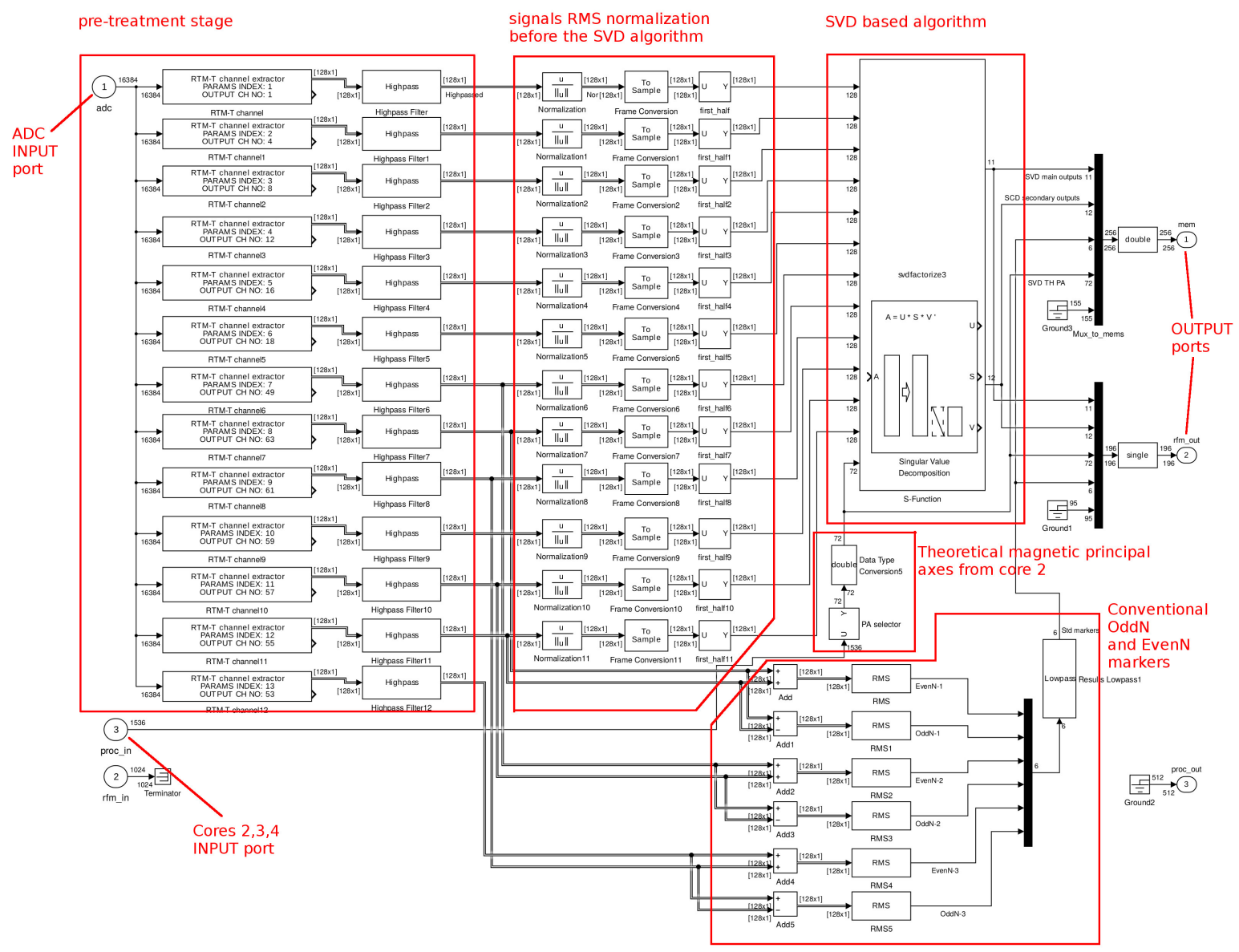

Fig. 6. Simulink diagram MHD analysis algorithm on node 7 core 1 .

algorithm that runs on core 2 is omitted for brevity. This Simulink block is contained in the wrapper of figure 5. In the algorithm model, we followed the usual Simulink convention with the signals flow from left to right. So the ADC input port is placed at top-left in the figure and supplies all acquired 64 channels, each having 256 samples. This data buffer is decomposed by the pre-treatment stage whose tasks are to extract 13 channels (12 for the SVD plus 1 for one of the conventional markers), to apply the inverse sensor-adc transfer function to compensate for the analog chain, $2 \mathrm{x}$ digital downsample the signals (applying a low pass filter and a $2 \mathrm{x}$ decimator) and to apply a high-pass filter. Pre-treated channels are prepared by the RMS normalization stage before being fed to the actual SVD factorization and post-processing algorithm (currently implemented as a Simulink S-function C++ block). The outputs of this block are passed to two output ports of the core: the RFM port to distribute the results to other nodes and the memory port to store the results on the MDSplus database for later analysis. The SVD factorization block needs the theoretical principal axes computed by the companion algorithm that runs on core 2. This data enters the block through the proc_in port at the bottom left of the figure, is extracted by a signal extraction block and connected to the SVD block.

Six pre-treated signals are employed to compute three pairs of conventional oddN and evenN markers. These signals come from three couples of fast magnetic sensors located each 180 degrees apart on the equatorial LFS plane on the machine (each couple at a different absolute toroidal angle). From their location, summing and subtracting their signals and cascading an RMS average stage (and possibly low pass filtering) provides information on the presence and amplitude of modes with even and odd toroidal mode number $(\mathrm{N})$ separately. This algorithm was installed and is depicted at the bottom right of figure 6.

\section{Tests On The TCV Plasma}

Node 7 has been tested on real TCV plasmas during the most recent campaign with the MHD analysis code described in section III-B. The node performed well both in terms of real time performance and effectiveness in providing on line MHD activity markers. Figure 7 shows real time traces measured by the hardware interface code on node 7 during a discharge. The abscissa axis is shot time, starts at $\mathrm{t}=-0.5 \mathrm{~s}$ and ends at $\mathrm{t}=2.0 \mathrm{~s}$ (breakdown is intended at $\mathrm{t}=0.0 \mathrm{~s}$ ). Timings are arranged by the MDSplus archiving routines of node 7 in order to facilitate algorithms' computational time compliance check by the system operator. In particular, timings $\mathrm{t} 2, \mathrm{t} 3, \mathrm{t} 4$ are plotted "upside-down"" from the cycle time (t1) track whereas cores' computational time are plotted normally. In this way it 


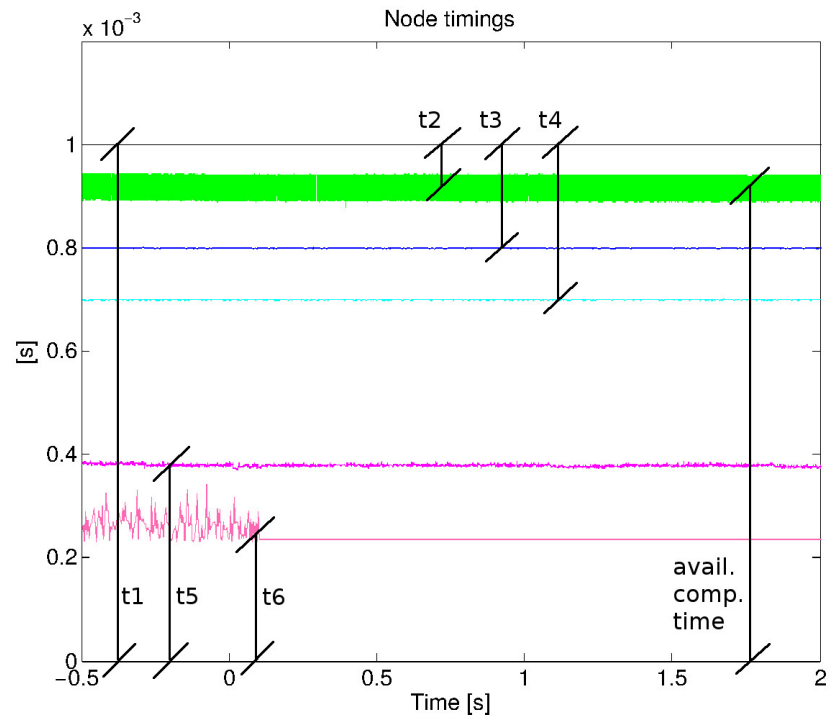

Fig. 7. Timing traces of node 7 during TCV shot no. 54642.

is simple to check when a core isn't able to keep-up with the RT requirements since the available computational time is quickly identified (see figure 7 on the right).

The node was parametrized with a $1 \mathrm{~ms}$ nominal cycle time and the measured one exactly matches with only some $\mu \mathrm{s}$ of jitter (t1 in figure 7). With two active processing cores data distribution takes approximately $80 \mu s$ ( 2 interval, in figure 7). The two processing cores execute the two algorithms in parallel and respect the limit on available computational time, as depicted by $\mathrm{t} 5$ and t6 time intervals in the figure. Finally reflective memory read and write synch timestamps are recorded ( $\mathrm{t} 3$ and $\mathrm{t} 4$ time intervals in the figure), proving that the $10 \mathrm{kHz}$ RFM strobe synch from RFM master node (node 2 in this shot) was correctly captured and data was correctly distributed to and from the real time network.

Figure 8 shows some MHD analysis results. The abscissa axis is identical to figure 7 . The first plot shows node timings as in figure 7 while the second plot shows raw ADC channels of magnetic signals from TCV. The third plot shows normalized SVD based NTM presence markers, as described in [14]. Briefly, the $\mathrm{H}$ marker is the entropy of the set of normalized singular values and is thus related to the degree of phase coherence shown by all the magnetic signals. The more it is near 0 the more we have a phase coherent signal in all the sensors and so one or more rotating modes. The P1 and P2 markers are the relative squared magnitude of the first and second couples of singular values w.r.t. all the others; their behavior is opposite w.r.t the $\mathrm{H}$ marker and they are linked to the presence of only one $(\mathrm{P} 1)$ or two $(\mathrm{P} 2)$ rotating modes at the same time. The fourth graph displays the frequency of the rotating mode, if any, and the fifth the $(2,1),(3,1)$ and $(3,2)$ modes likelihood markers. The last plot shows the standard MHD markers oddN and evenN. The interested reader is pointed to [14] for further information on this algorithm.

Looking at these signals, this shot is characterized by a quick $(3,1)$ rotating mode just after the breakdown and by a
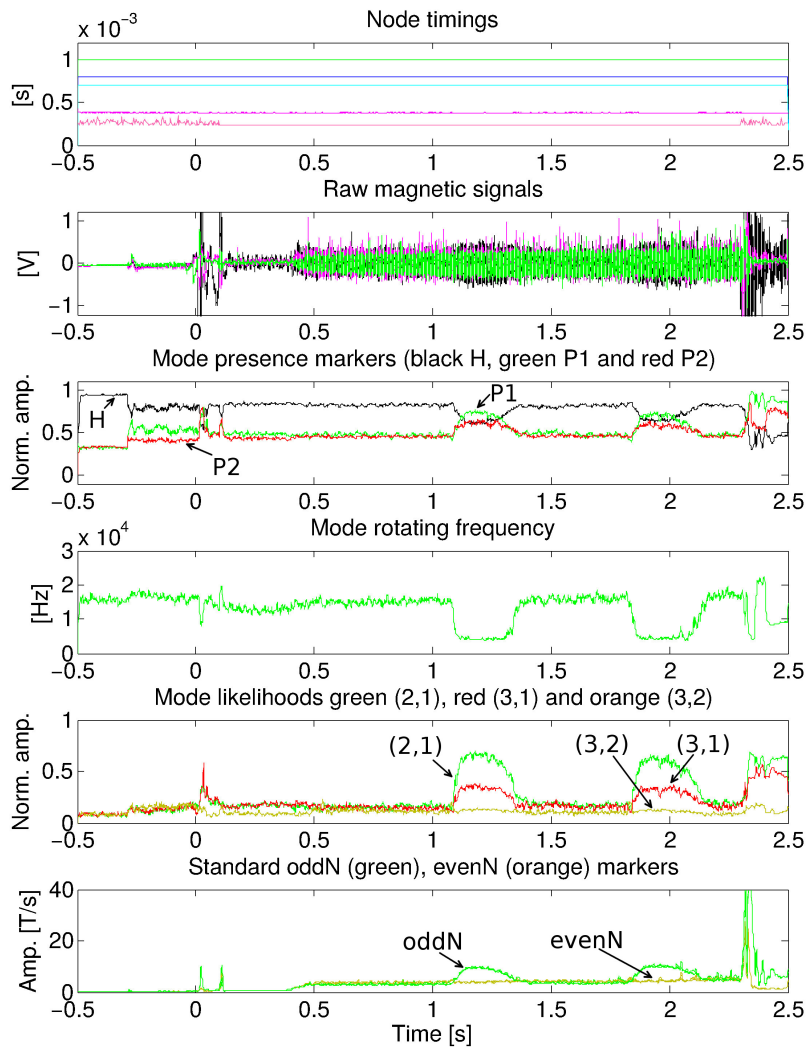

Fig. 8. MHD analysis results during TCV shot no. 54642.

$(2,1)$ mode in the interval $t=[1.1,1.4]$ and again in the interval $\mathrm{t}=[1.8,2.2]$. Since these signals are distributed on the real time network, they can be used to trigger countermeasures to control the MHD activity (for instance by means of ECR heating) or, more simply, to soft land the discharge without incurring a full high current disruption. For example, in the presented shot, ECRH beams were switched on triggered by the $(2,1)$ likelihood signal and effectively compensated the rotating mode at $\mathrm{t}=1.4$ and at $\mathrm{t}=2.2$.

\section{Conclusion And Outlook}

We have described the integration of a new node into the TCV real time control system. The new node is tailored to performing advanced analysis algorithm on the fast magnetic signals of TCV in real time and to distribute the results to the real time network of the control system. During the development of the system we succeeded in respecting all useful features of the legacy system, namely: a strict separation between the environment in which algorithms are developed (Mathworks/Simulink) and that in which they are executed (custom $\mathrm{C} / \mathrm{C}++$ code) and compatibility with the legacy data interface on the RFM network, etc. New features have also been introduced: a real-time packet acquisition and processing approach, multi-core data processing and data transfers on a multi synchronous system (ADC side and RFM side). 
We think that the approach followed in this paper can be continued in the future whenever there is the need for the realtime processing of fast diagnostics with complex algorithms and to distribute results to other actors in the control system. One example on our system is the soft-x XTOMO acquisition node no. 5, which could be refurbished with an approach like that described here.

\section{ACKNOWLEDGMENT}

The authors are grateful to the TCV team, which provided great help in commissioning the described system.

\section{REFERENCES}

[1] Nardone, C. (1992). Multichannel fluctuation data analysis by the singular value decomposition method. Application to MHD modes in JET. Plasma Phys. Control. Fusion. 34, 1447.

[2] Dudok de Wit, T. (1994). The biorthogonal decomposition as a tool for investigating fluctuations in plasmas. Phys. Plasmas. 1, 3288.

[3] Schittenhelm, M. (1997). Analysis of coupled MHD modes with Mirnov probes in ASDEX Upgrade. Nucl. Fusion. 37 1255-70.

[4] Kim, J. S. (1999). MHD mode identification of tokamak plasmas from Mirnov signals. Plasma Phys. Control. Fusion. 41, 1399-420

[5] Paley, J. I. (2010). Architecture and commissioning of the TCV distributed feedback control system. Presented at 17th IEEE-NPSS 2010, pp. 1-6.

[6] Felici, F. (2014). Development of real-time plasma analysis and control algorithms for the TCV tokamak using Simulink. Fusion Eng. Design. $89,165-176$.

[7] Le, H. B. (2014). Distributed digital real-time control system for TCV tokamak. Fusion Eng. Design. 89, 155-164.

[8] Abaco Systems (a General Electric's spin-off company) PCIE-5565PIORC board product page. [Online]. Available: https://www.abaco.com/products/pcie-5565piorc

[9] Mathworks Simulink product introduction page. [Online]. Available: http://www.mathworks.com/products/simulink/

[10] Mathworks Simulink Embedded Coder product page. [Online]. Available: http://www.mathworks.com/products/embedded-coder/

[11] Galperti, C. (2014). Testing and commissioning the multinode ECRH realtime control system on the FTU tokamak. Fusion Eng. Design. 89, 214-223.

[12] D-TACQ Solutions ACQ196 acquisition system product page. [Online]. Available: http://www.d-tacq.com/acq196cpci.shtml

[13] Intel's TSC technology introduction on Wikipedia. [Online]. Available: https://en.wikipedia.org/wiki/Time_Stamp_Counter

[14] Galperti, C. (2014). Development of real-time MHD markers based on biorthogonal decomposition of signals from Mirnov coils. Plasma Phys. Control. Fusion. 56, 114012. 